\title{
The CPMDS catalogue of common proper motion double stars in the Bordeaux Carte du Ciel zone ${ }^{\star}$
}

\author{
P. Gavras ${ }^{1,2}$, D. Sinachopoulos ${ }^{2}$, J. F. Le Campion ${ }^{3,4}$, and C. Ducourant ${ }^{3,4}$ \\ 1 Department of Astrophysics, Astronomy and Mechanics, Faculty of Physics, University of Athens, 15783 Athens, Greece \\ e-mail: pgavras@astro.noa.gr \\ 2 Institute of Astronomy \& Astrophysics, National Observatory of Athens I. Metaxa \& Bas. Pavlou GR. 15236 Palea Penteli, Greece \\ e-mail: ds@astro.noa.gr \\ ${ }^{3}$ Université de Bordeaux, Observatoire Aquitain des Sciences de l’Univers, 2 rue de l'Observatoire, BP 89, 33271 Floirac Cedex, \\ France \\ e-mail: Christine.Ducourant@obs.u-bordeaux1.fr \\ 4 CNRS, UMR 5804, Laboratoire d'Astrophysique de Bordeaux, 2 rue de l'Observatoire, BP 89, 33271 Floirac Cedex, France
}

Received 29 September 2009 / Accepted 8 June 2010

\section{ABSTRACT}

\begin{abstract}
Context. The present knowledge of common proper motion double stars is rather poor and almost entirely restricted to the Luyten LDS catalogue of 6210 pairs, to 439 pairs of Halbwachs catalogue of common proper motion stars in AGK3, and to Greaves new northern common proper motion pairs (975 pairs).

Aims. We present a search for new double stars with common proper motion in the Bordeaux, PM2000, proper motion catalogue $\left(+11^{\circ} \leq \delta \leq+18^{\circ}\right)$.

Methods. The selection of double stars, on the basis of common proper motion, was performed by testing the candidate pairs with a Student t-distribution hypothesis test. A classification was performed by the analysis of the photometric properties of the components. Results. We present a catalogue, which contains 2572 new common proper motion pairs with angular separations $\rho \leq 2^{\prime}$ and PM2000 meridian magnitudes for both components. As a by-product, we present the remeasurement of the parameters of 926 visual pairs from the WDS Washington double star catalogue.

Conclusions. The present catalogue, which is a list of candidate binaries, is an important step in the knowledge of double stars because it increases the number of known common proper motion pairs by $30 \%$.
\end{abstract}

Key words. binaries: visual - astrometry - catalogs

\section{Introduction}

The Bordeaux PM2000 catalogue (Ducourant et al. 2006) is a project that started in 1998 at Bordeaux observatory and finished in 2006, providing accurate measurements of proper motions and positions for stars brighter than $V=16.2 \mathrm{mag}$. It provides measurements for more than 2600000 stars (complete to $V=15.4$ ) located in the Bordeaux Carte du Ciel zone $\left(+11^{\circ} \leq \delta \leq+18^{\circ}\right)$

The project used observations of 5 different catalogues indicated in Table 1 with the mean epoch of observations. The Bordeaux meridian positions used, were published as the catalogue M2000 (Rapaport et al. 2001). The Bordeaux meridian circle has $202 \mathrm{~mm}$ diameter of front lens and uses a Thomson $7896 \mathrm{M}$ CCD camera with dimensions $1024 \times 1024$ pixels. The size of each pixel is $19 \times 19 \mu \mathrm{m}$ and corresponds to $1.65 \times$ 1.65 arcsec (Viateau et al. 1999). The epoch of the first observations used for PM2000 is the epoch of the Carte du Ciel plates $(\sim 1900)$ and the last epoch is that of M2000 ( 2000) (Ducourant et al. 2006).

In this present work, we attempt to identify the common proper motion (hereafter CPM) visual double stars in PM2000,

* Complete Tables 2-4 are only available in electronic form at the CDS via anonymous ftp to cdsarc.u-strasbg.fr $(130.79 .128 .5)$ or via

http: //cdsarc.u-strasbg.fr/viz-bin/qcat?J/A+A/521/A4
Table 1. PM2000 data sources.

\begin{tabular}{lll}
\hline \hline Source & Author & Epoch \\
\hline AC2000.2 & Urban et al. 1998 & $\sim 1900$ \\
CdC2000 & Rapaport et al. 2006 & $\sim 1900$ \\
USNO-A2.0 & Monet et al. 1998 & $\sim 1950$ \\
Yellow Sky (YS3.0) & Unpublished & $\sim 1978$ \\
M2000 & Rapaport et al. 2006 & $\sim 2000$ \\
\hline
\end{tabular}

lying in the Bordeaux Carte du Ciel zone and to provide measurements of their angular separation and position angle.

Much work has been done in the field of double stars, trying to discriminate the physically related pairs, from the opticals. The first approach was that of Aitken (1911) who adopted a criterion for which a double star would be physical, taken into account the angular separation and the combined magnitude of the components. Kubikowski et al. (1959) published a statistical study of the topic. Sinachopoulos and Mouzourakis (1991) proposed a statistical criterion to discriminate the physical visual double stars from the optical ones.

The use of proper motions of the components to identify the physically related pairs among the visuals was firstly presented by Luyten (1933). The LDS common proper motion double star catalogue (Luyten 1997) contains 6210 pairs. Another major catalogue was presented by Halbwachs (1986) containing 439 double stars with common proper motion in the AGK3 catalogue. 
A by-product of the present work is to provide new relative coordinates of the known visual double stars, which are present in PM2000. There are currently, two major catalogues of visual double stars. One is the Catalogue of Components of Double \& Multiple stars (CCDM), which contains 105838 double stars (Dommanget \& Nys 2002). The other is the Washington Double Star catalogue (WDS) containing 102387 double and multiple stars (Mason et al. 2006).

In Sect. 2 of this paper, we present a search for common proper motion double stars in PM2000 and the properties of the resulting catalogue. In Sect. 3, we search for the known WDS double stars, which are present in PM2000. In Sect. 4, we present our results as the Common Proper Motion visual Double Star catalogue (CPMDS), an annex of high proper motion and large angular separation pairs, and a catalogue with remeasurements of known WDS double stars.

\section{Searching for common proper motion stars in PM2000}

\subsection{Selection criterion}

We first extracted from PM2000, all stars with proper motions larger than $3 \sigma$ in both directions. By doing this, we selected 414224 stars. Since about $97 \%$ of the WDS entries have angular separations smaller than $2^{\prime}$, we chose this constrain, to check whether a star in our PM2000 subsample has one or more neighbouring stars at angular separation less than or equal to $2^{\prime}$. The result was a sample of 213215 visual double stars.

We then, checked which of these double stars are common proper motion pairs. To do that, we carried out the following statistical hypothesis test. We considered the primary component of a double star with proper motion $\mu_{1 \alpha} \cos \delta_{1}$ (hereafter $\mu_{1 \alpha}^{*}$ ), with an unbiased estimation of the variance $S_{\mu_{1 \alpha} \cos \delta_{1}}$ (from now on $S_{\mu_{1 \alpha}^{*}}$ ) and with an associated $\mu_{1 \delta}, S_{1 \delta}$ derived from $n_{1 \alpha}$ and $n_{1 \delta}$ observations. The secondary component parameters are $\mu_{2 \alpha}^{*}, S_{\mu_{2 \alpha}^{*}}$, and $n_{2 \alpha}$ and $\mu_{2 \delta}, S_{2 \delta}$, and $n_{2 \delta}$ respectively. We verified whether the proper motions in both directions are the same at the 0.05 level of significance.

Fewer than 30 measurements were typically used for each PM2000 star to calculate its proper motion. For this reason, we had to use a test based on the Student's t-distribution. For stars, which are observed more than 30 times, the normal distibution should be used, but Student's t-distribution becomes the normal distribution when the degrees of freedom are more than 30 . The null and alternative hypotheses of our t-test were

$H_{0 \alpha}: \mu_{1 \alpha}^{*}=\mu_{2 \alpha}^{*}$

$H_{1 \alpha}: \mu_{1 \alpha}^{*} \neq \mu_{2 \alpha}^{*}$

$H_{0 \delta}: \mu_{1 \delta}=\mu_{2 \delta}$

$H_{1 \delta}: \mu_{1 \delta} \neq \mu_{2 \delta}$

To solve this problem, we had to calculate the $T_{\alpha}$ variable for right ascension

$T_{\alpha}=\frac{\mu_{1 \alpha}^{*}-\mu_{2 \alpha}^{*}}{\sigma_{\alpha} \sqrt{\frac{1}{n_{1 \alpha}}+\frac{1}{n_{2 \alpha}}}}$

where

$\sigma_{\alpha}=\sqrt{\frac{\left(n_{1 \alpha}-1\right) S_{\mu_{1 \alpha}^{*}}^{2}+\left(n_{2 \alpha}-1\right) S_{\mu_{2 \alpha}^{*}}^{2}}{n_{1 \alpha}+n_{2 \alpha}-2}}$ and similarly the $T_{\delta}$ variable for declination. We calculated the values $T_{\alpha}$ and $T_{\delta}$, of t-distribution for $n_{1 \alpha}+n_{2 \alpha}-2$ degrees of freedom and 0.05 , level of significance. To accept the null hypotheses, the expressions that should be valid are

$\left|T_{\alpha}\right| \leq T$

$\left|T_{\delta}\right| \leq T$

where $T$ is the value for a 0.05 level of significance depending on the degrees of freedom.

We calculated $T_{\alpha}$ and $T_{\delta}$ and $T$ only for those pairs, for which both stars in our selected sample of PM2000 stars had more than 3 observations used to derive their proper motions. If both conditions expressed in Eqs. (7) and (8) found to be true, we decided that the components of this double star had common proper motion. We also searched for CPM stars in pairs with $\mu>50 \mathrm{mas} / \mathrm{yr}$ and $2^{\prime}<\rho \leq 5^{\prime}$, using the same t-test described previously. We also calculated the probability $\beta$ of commiting a type II error, which is the probability of accepting a pair as having CPM, although the proper motions of its components are different. This probability is represented by the probabilities $\beta_{\alpha}$ in $\alpha$ and $\beta_{\delta}$ in $\delta$. To calculate $\beta_{\alpha}$, we had to calculate first the critical value of the difference in the proper motions $\Delta \mu_{\alpha}^{*}$ for right ascension

$\Delta \mu_{\alpha}^{*}=T \sigma_{\alpha} \sqrt{\frac{1}{n_{1 \alpha}}+\frac{1}{n_{2 \alpha}}}$.

Afterwards, we calculated the standardized variable $T_{\beta_{\alpha}}$, which is given by the equation

$T_{\beta_{\alpha}}=\frac{\mu_{1 \alpha}^{*}-\mu_{2 \alpha}^{*}-\Delta \mu_{\alpha}^{*}}{\sigma_{\alpha} \sqrt{\frac{1}{n_{1 \alpha}}+\frac{1}{n_{2 \alpha}}}}$

Obviously the probability $\beta_{\alpha}$ of commiting a type II error in $\alpha$ is the area covered by the $\mathrm{t}$-distribution up to $T_{\beta_{\alpha}}$.

We followed the same procedure to calculate $\beta_{\delta}$ as well. The global solution performed to derive the proper motions in PM2000 was performed at a selected central epoch for which the matrix of error is diagonal. Therefore the two components of proper motion are independent. Thus, the total probability is given by

$\beta=\beta_{\alpha}+\beta_{\delta}-\beta_{\alpha} \beta_{\delta}$

\subsection{Properties of our common proper motion double star sample}

We found 2572 common proper motion double stars with angular separations smaller than $2^{\prime}$ and 259 with angular separations between $2^{\prime}$ and $5^{\prime}$, but with proper motions larger than 50 mas/yr. Among these, 2831 CPM stars 53 are triples and 6 are multiple systems. Sixty-four pairs are members of the open cluster M 67.

In Fig. 1, we show the distribution of the ratio of the number of CPM pairs found to the total number of visual pairs of our sample $\left(\rho \leq 2^{\prime}\right)$. As we see, the ratio for CPM double stars is higher for small angular separations. In the range $5^{\prime \prime} \leq \rho \leq 10^{\prime \prime}$, the calculated ratio is underestimated because the lower limit of separation in PM2000 is about $8^{\prime \prime}$. In the inserted diagram, we present the distribution of the absolute number of CPM double stars.

In Fig. 2, we present the distribution of the errors of the angular separation for $\rho \leq 2^{\prime}$. The mean value of error in angular separation is $0.06^{\prime \prime}$. The mean value of error in the measurement 


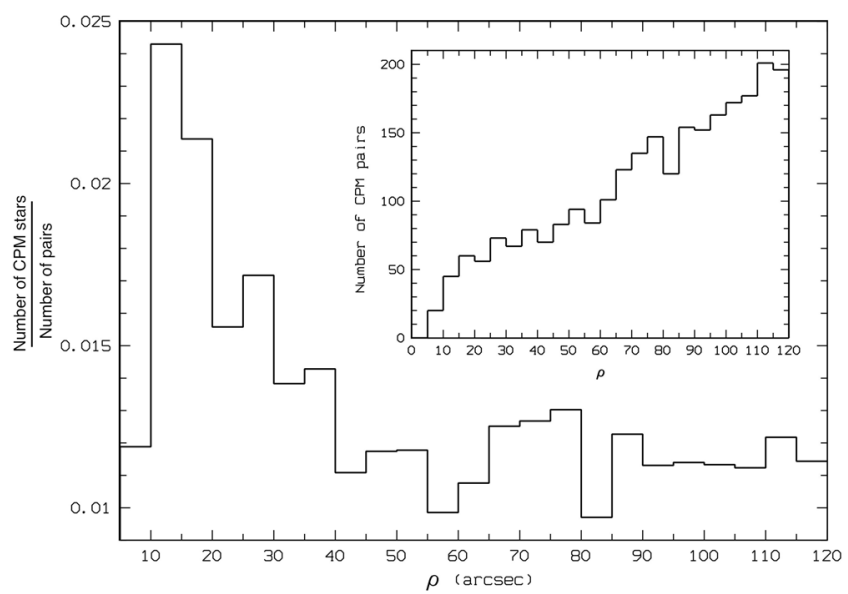

Fig. 1. The distribution of $\rho$ of the ratio of the number of common proper motion stars to the total number of double stars in our sample. Insert: The distribution of the angular separation of the common proper motion stars with $\rho \leq 2^{\prime}$.

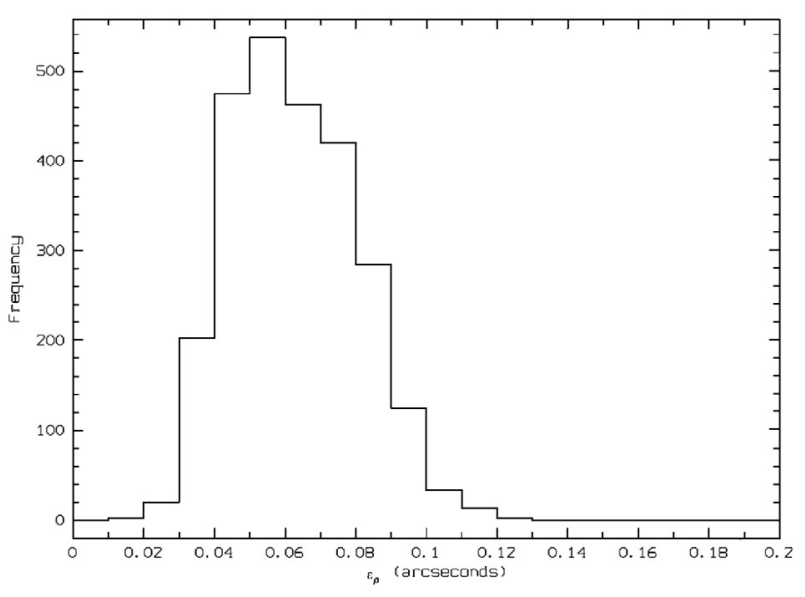

Fig. 2. The distribution of the measurement errors in $\rho$ for $\rho \leq 2^{\prime}$.

of position angle is $0.06^{\circ}$. In the case of $2^{\prime}<\rho \leq 5^{\prime}$, the mean value of errors are $0.07^{\prime \prime}$ and $0.02^{\circ}$, respectively.

In Fig. 3, we present the distribution of the probability $\beta$ for the pairs with angular separations smaller than $2^{\prime}$. About $33 \%$ of these pairs have a probability of less than $10 \%$ of not being common proper motion double stars despite being selected as CPM ones, while $7 \%$ of them have $\beta$ higher than $50 \%$.

In the case of $2^{\prime}<\rho \leq 5^{\prime}$, the probability $\beta$ is less than $10 \%$ for about $38 \%$ of the double stars, while for $8 \%$ of these pairs the probability of commiting a type II error is $50 \%$ or higher. These are rather optimistic results, since the type II error is underestimated due to the solar motion to the apex, which was not taken into account.

\subsection{External check of our CPM selection}

To test our results, we first selected the objects of Luyten's LDS common proper motion double star catalogue, which are in the Bordeaux zone. We, then excluded very faint stars and pairs with angular separations smaller than $7^{\prime \prime}$, since they could not be included in our lists. Afterwards, we identified the remaining LDS stars in PM2000. We, finally found 64 Luyten CPM stars in PM2000. Only 12 of them (19\%) were identified by us as CPM

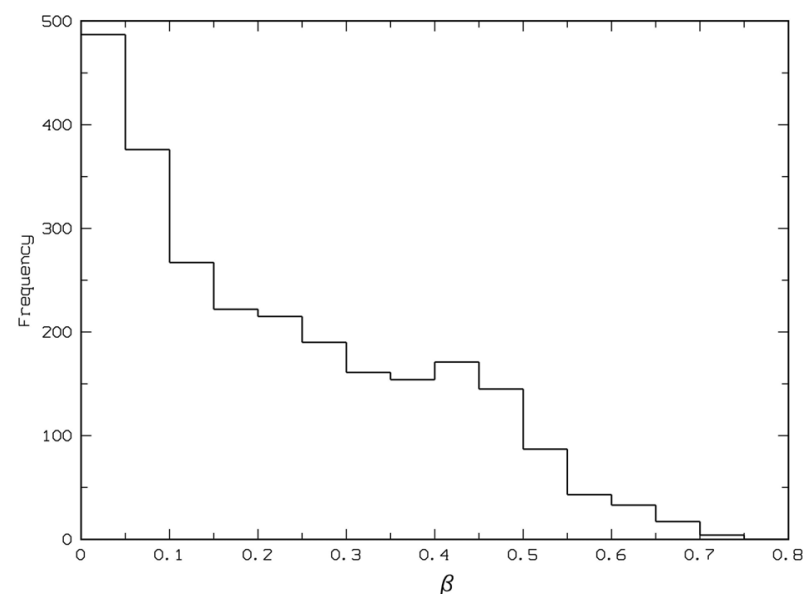

Fig. 3. The distribution of the probability $\beta$ to commit a type II error for $\rho \leq 2^{\prime}$.

double stars. For the 42 missed pairs, the discrepancy between LDS and our list may be caused by a too severe selection criterion in this work. We reapplied our test, for a significance of 0.01 and we were able to accept as CPM pairs 10 more of the 42 missed. Nevertheless, we were unable to accept as CPM half of the LDS pairs.

The origin of this discrepancy cannot be the quality of the proper motions used in the present work, because we searched both in PPMX (Roeser et al. 2008) and UCAC2 (Zacharias et al. 2004) and found their proper motions to be fully compatible with those of PM2000. Therefore the final explanation of the 32 discrepant pairs is that Luyten's criterion is more tolerant than ours.

\subsection{Classification of our CPM sample}

To identify and validate the pairs that could possibly have a physical connection, we used Vizier, where we searched the 2MASS catalogue (Cutri et al. 2003) for photometry of both components of our CPM pairs. From the 2831 double stars in our lists, we found 2MASS high quality data for both components only for 2603 of them. We then created an empirical $H-R$ diagram $(J-K, J)$ using data of the old open cluster M67 and Pleiades, taking into account that these clusters have almost the same reddening. The value of $E(B-V)$ is indeed, $41 \pm 4$ mmag for M 67 (Taylor 2007) and $57 \pm 5.8 \mathrm{mmag}$ for the Pleiades (Taylor 2008). Moreover, we used the near infrared bands of 2MASS, so the effect of reddening is less important than it is in optical wavelengths. The distance of M 67 is 843 pc (Tian Kai-ping et al. 1992), while the distance of Pleiades is $133.8 \mathrm{pc}$ (Percival et al. 2005); we used these values to construct the common $H-R$ diagram of these two clusters. Our idea of validating double stars is that physically connected pairs should have about the same age and distance and take specific relative places on an $H-R$ diagram depending on the initial mass of their components.

We classified our CPM sample according to the likehood that their components are physically connected. Each pair was considered in this $H-R$ diagram and classified as $I, I I, I I I, I V$ and $V$. The double stars with components of similar $(J-K)$ and magnitudes were considered members of class I. The pairs with secondaries that are on the main sequence (MS), while their primary is on either the MS or the giant branch were also considered as members of class I. On the other hand, double stars with the secondary component brighter in $J$ were classified as members of 


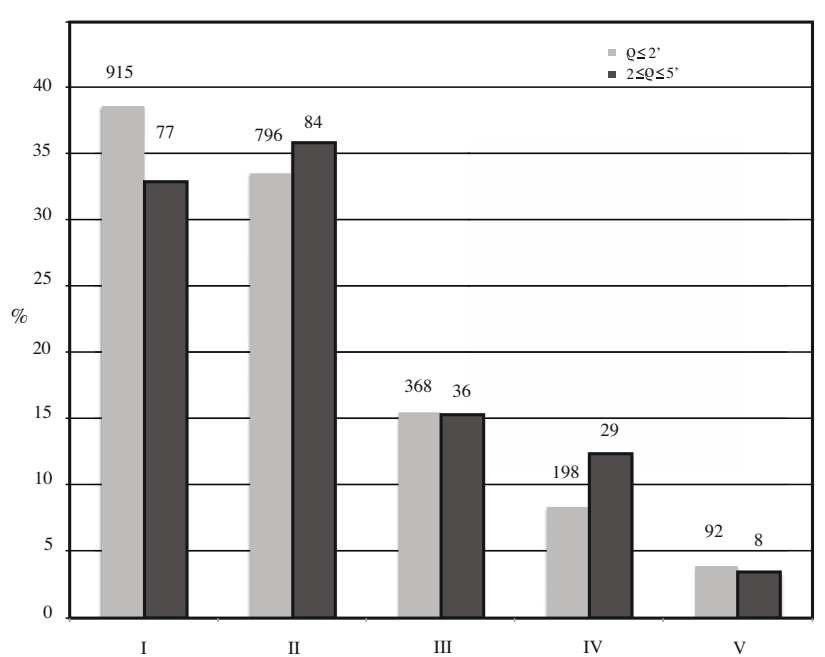

Fig. 4. Distribution of the classes of the common proper motion stars in percentage with $\rho \leq 2^{\prime}$ and $2^{\prime}<\rho \leq 5^{\prime}$. On top of every class we give corresponding number of pairs.

class V. In addition, pairs with a secondary much redder than the primary and of very similar magnitude, were classified as members of class $\mathrm{V}$, as long as they were not both on the giant branch. The pairs that we could not classify were considered as members of class III. Class II contains the cases between I and III, while class IV includes those between III and V. From the 2603 CPM double stars that we were able to check, 992 pairs were classified as members of class I, 880 as class II, 404 as class III, 227 double stars as class IV, and 100 as class V.

Since we have two data sets, one of angular separation smaller than $2^{\prime}$ and the other with $2^{\prime}<\rho \leq 5^{\prime}$, we verified whether the two distributions of our classification of stars are coherent. In Fig. 4, we present these two distributions. We note that class I pairs are slightly more frequent in the small angular separation sample, which is reasonable. At the same time, we found a higher percentage of class IV in the large angular separation list, which is also what we might expect. We also studied the distribution of the proper motions $\mu$ of those 2603 pairs for every class, but no correlation could be detected.

In Fig. 5, we present the distribution of $\rho$ for the pairs classified as I and II. We also applied a linear regression fit to this distribution for angular separations greater than $40^{\prime \prime}$. All the pairs lying above the trendline are considered to be genuine binaries (Halbwachs 2007). The rate of these genuine binaries over all pairs at angular $\rho \leq 40^{\prime \prime}$ is $39.8 \%$.

We were, finally able to compile a catalogue of 2572 new common proper motion double stars and classify them empirically according to the position of their components on the $H-R$ diagram. To this list, we add an annex of 259 common proper motion pairs with high proper motion $(\mu>50 \mathrm{mas} / \mathrm{yr})$ and large angular separation $2^{\prime}<\rho \leq 5^{\prime}$.

\section{Remeasurements of WDS double stars in PM2000}

We first extracted all known WDS double and multiple stars in the Bordeaux Carte du Ciel zone within the observing capabilities of the Bordeaux transit instrument. For instance, most of the double stars in WDS are bright and for these stars the lower limit to the angular separation in PM2000 is $8^{\prime \prime}$. Using these restrictions, we obtained a preliminary list of 1500 WDS double stars that could have been found in PM2000. To identify

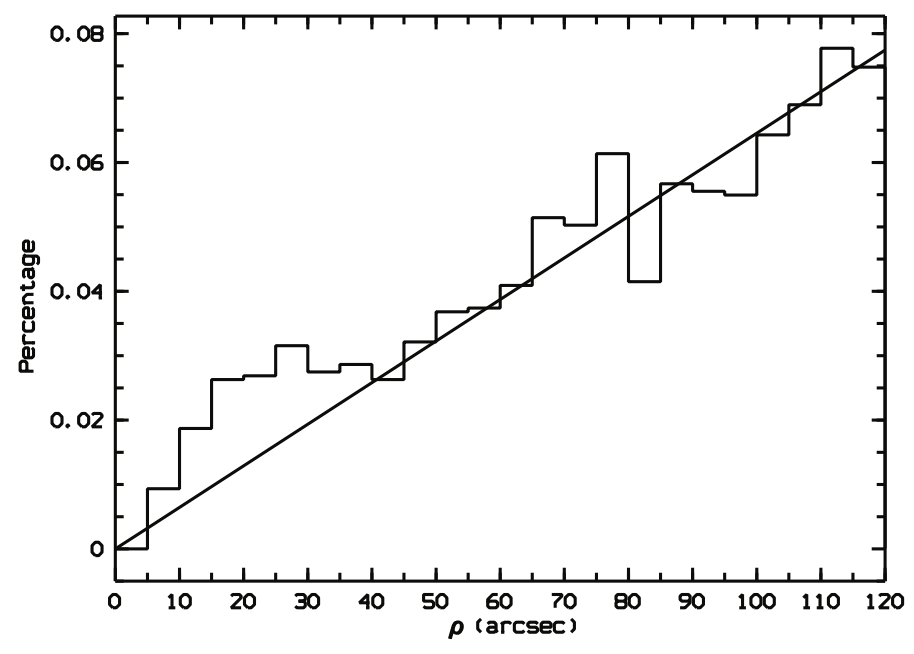

Fig. 5. Distribution the angular separation of the classes I and II with $\rho \leq 2^{\prime}$. The line corresponds to the fitting of the distribution of the pairs with $\rho \geq 40^{\prime \prime}$.

these stars in the PM2000 catalogue and extract their data, we searched for stars in common between these two catalogues by cross-correlating their coordinates and magnitudes. The criterion adopted to establish that a star is common to both catalogues is that the maximum difference in $(\alpha, \delta)$ is at most $3^{\prime \prime}$. Although our software was designed to perform also a cross-checking of the magnitudes, this criterion had to be discarded because of the different photometric techniques used in the two catalogues.

In the first step, we attempted to find the primary component. Once a WDS primary component was found in PM2000, the coordinates of its secondary were calculated, using the information of angular separation $\rho$ and the position angle $\theta$ of the WDS double star as well as the position $(\alpha, \delta)$ of the primary star in PM2000. From these values, the expected position of the secondary component was calculated and using the same procedure and constraints applied to the primaries, we searched for the secondary component in the PM2000 catalogue.

Using our initial list of 1500 WDS double stars, we found 1442 WDS primaries $(96.2 \%)$, but identified both components in only 1287 cases (89.3\%). Thirty-seven of those 1287 secondaries, were found manually using images of DSS for comparison. These 37 stars were found to have angular separations that differ by more than $5^{\prime \prime}$ from the values given in WDS. The reason for this difference is that most of these double stars have not been observed since 1950. Thus, the optical pairs among them, which have relatively large differences in the proper motions of their components, had values of $(\rho, \theta)$ in PM2000, which differ from the values listed in WDS.

In the next step, we gathered all the Bordeaux meridian CCD observations of the components of these double stars, which were used in the preparation of PM2000. We handled only those pairs for which more than 3 measurements per component were available. We finally obtained a list of 1000 double stars for which we were able to calculate angular separation, position angle, and magnitude of the components for the mean epoch of observations.

In some of the double stars in our list (especially these with relatively large angular separation), there are some observations used to compile PM2000 for which we could indentify either the primary or the secondary stars. In these cases, we calculated $(\rho, \theta)$ for the mean epoch using the observations of different epochs for each component only if the difference in the epochs 

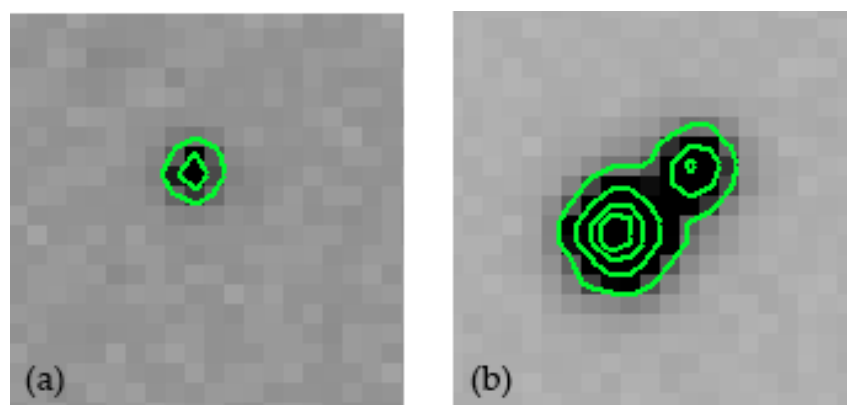

Fig. 6. Examples of unidentified double stars. a) WDS $12472+1442$. Only the primary component was detected. b) WDS $13012+1552$ with angular separation 8.4" not resolved by the Bordeaux Transit Instrument.

of the two components was shorter than six months. This method delivers reliable relative coordinates of the double stars because the angular separation and position angle of very wide double stars hardly change from year to year.

The result is a catalogue of 926 WDS double stars containing $(\rho, \theta)$ for an epoch of about 2000, with mean precisions $\sigma_{\rho}=$ $0.026^{\prime \prime}$ and $\sigma_{\theta}=0.06^{\circ}$. Only 24 of them $(2.6 \%)$ are included in our CPMDS list.

We were unable to identify both components for 213 WDS double stars from a preliminary list of 1500 WDS pairs $(16 \%)$. We checked all these double stars one by one. In most cases, the secondary star was too faint. Figure 6a shows a CCD frame of the Bordeaux meridian circle of a double star with a $13.47 \mathrm{mag}$ primary and a 15.8 mag secondary. The secondary component obviously, could not be detected in this frame. In many other cases, the WDS double star was unresolved (Fig. 6b). In some other cases, the last WDS measurement was derived 50 years ago or even earlier. Optical pairs observed so many years ago that have components with large and different proper motions, have angular separations and position angles that vary so much that it is impossible to identify them in more recent catalogues. 287 pairs had less than 3 observations of their components and 74 pairs of the identified double stars had poor astrometric results, and were rejected.

In Fig. 7 we present the distribution $\Delta \rho$, which is the difference of our calculated $\rho$ minus the last observation listed in WDS. This distribution is restricted to the case of $-1^{\prime \prime}<$ $\rho_{\text {PM2000 }}-\rho_{\text {WDS }}<1^{\prime \prime}$. The mean value of $\Delta \rho$ for these stars is $0.013^{\prime \prime}$ with $\sigma=1.05^{\prime \prime}$. We present the whole distribution in the insert. These results are in good agreement with the measurements of WDS.

\section{Presentation of the catalogues}

\subsection{The CPMDS common proper motion double star catalogue}

Our CPMDS catalogue contains all detected common proper motion double stars in the Bordeaux zone. The main catalogue contains 2572 CPM double stars with angular separations smaller than $2^{\prime}$. Its annex presents 259 common proper motion pairs with proper motions greater than $50 \mathrm{mas} / \mathrm{yr}$ and angular separations larger than $2^{\prime}$ and smaller than $5^{\prime}$. An extract of the main catalogue and its annex is given in Tables 2 and 3, respectively. Both catalogues are available at the CDS. The first two columns give the $\mathrm{J} 2000$ coordinates of the primary component. The next column is the identifier of PM2000 for the primary and the secondary component. Columns 4 and 5 give the angular

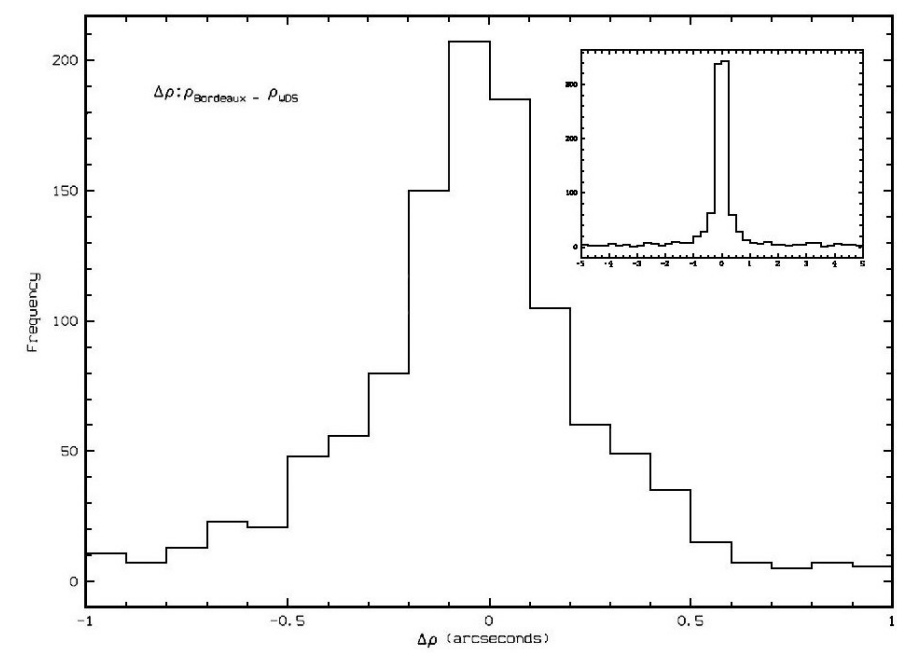

Fig. 7. The central part of the distribution of our angular separation minus the angular separation from WDS. The mean value of $\Delta \rho$ for these stars is $0.013^{\prime \prime}$ with $\sigma=1.055^{\prime \prime}$. In the inserted picture, we present the whole distribution

separation and position angle, respectively. We show in Col. 6, the PM2000 magnitudes of the primary and the secondary component, respectively. In Col. 7 we give the PM2000 proper motion of the primary and in Col. 8 its number of observations used in PM2000 for the calculation of the proper motion in $\alpha$ and $\delta$. In Col. 9 we give the proper motion of the secondary stars and in Col. 10 the number of observations used in the calculation of the proper motion in $\alpha$ and $\delta$. In Col. 11, we indicate the central epoch of observations and give our classification according to the position on the empirical $H-R$ diagram in Col. 12. Finally, in Col. 13 , we present the probability $\beta$ of committing a type II error, which is to accept as CPM a pair with random proper motions.

\subsection{The catalogue of remeasured parameters of known WDS double stars}

We present an extract of the remeasured parameters of known WDS stars in the Bordeaux zone in Table 4. The full catalogue is also available at the CDS. The first two columns show the J2000 coordinates of the primary component. The third column indicates its WDS identification. In Col. 4, we present the calculated angular separation and its error. In Col. 5, we list the calculated position angle with its error. Column 6 contains the magnitudes of the primary and the secondary components given in PM2000. In Col. 7, we give the mean epoch of observation and finally in Col. 8 the number of observations used.

\section{Conclusions}

In the proper motion catalogue PM2000, we have identified a sample of new common proper motion double stars (CPMDS) by selecting only the highest quality proper motion measurements of the catalogue and conducting a t-test. The result was the CPMDS catalogue with 2572 pairs and its annex with 259 double stars with $2^{\prime}<\rho \leq 5^{\prime}$ and proper motions larger than 50 mas/yr. Our two lists are a useful tool in our quest to identify true binaries, as they provide a large number of binary candidates, waiting to be confirmed by radial velocity measurements. 

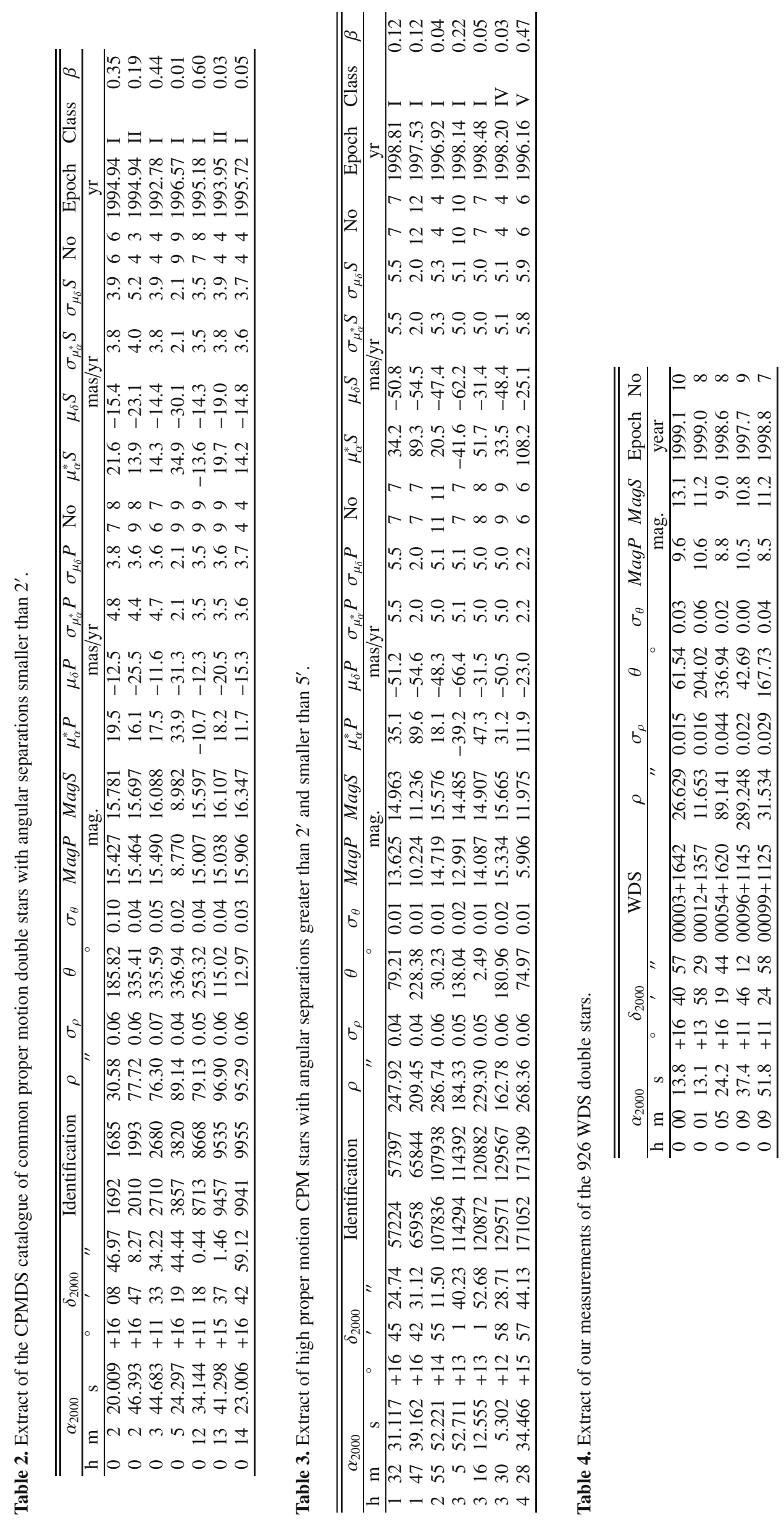
P. Gavras et al.: The CPMDS catalogue of common proper motion double stars in the Bordeaux Carte du Ciel zone

The area covered by the proper motion catalogue corresponds to about $6 \%$ of the total surface of the celestial sphere. In this $6 \%$, we discovered 2572 new double stars with common proper motion and angular separations smaller than $2^{\prime}$, which means that in the whole sky we expect about 43500 CPM double stars for this magnitude range.

Using positions of the stars of the same age in an empirical $\mathrm{H}-\mathrm{R}$ diagram we were able to estimate that about $72 \%$ of the pairs selected with our method belong to classes I and II. We were unable to classify $16 \%$ of the double stars and the remaining $12 \%$ were members of classes IV and V.

We also used the Bordeaux PM2000 catalogue to remeasure the parameters of known double stars from WDS of 926 WDS double stars. Only 24 of them have an entry in our CPMDS catalogue.

Acknowledgements. We thank the referee, Dr. J.L. Halbwachs, for helpful comments which led to an improved paper. This research has made use of the Washington Double Star Catalogue maintained at the US Naval Observatory. This research has made use of the VizieR catalogue access tool, CDS, Strasbourg, France. This publication makes use of data products from the Two Micron All Sky Survey, which is a joint project of the University of Massachusetts and the Infrared Processing and Analysis Center/California Institute of Technology, funded by the National Aeronautics and Space Administration and the National Science Foundation. This research is based on data mining of the Digitized Sky Survey, developed and operated by the Catalogs and Surveys Branch of the Space Telescope Science Institute, Baltimore, Maryland.

\section{References}

Aitken, R. G. 1911, Astron. Nachr., 188,281

Cutri, R. M., Skrutskie, M. F., van Dyk, S., et al. 2003, 2MASS All-Sky Catalog of Point Sources, NASA/IPAC Infrared Science Archive

Dommanget, J., \& Nys, O. 1994, Components of double and multiple stars (CCDM), 2002yCat.1269....0D

Ducourant, C., Le Campion, J. F., Rapaport, M., et al. 2006, A\&A, 448, 1235

Greaves, J. 2004, MNRAS, 355, 585

Halbwachs, J. L. 1986, A\&ASS, 66, 131

Halbwachs, J. L. 2007, Binary stars, ELSA School on the Science of Gaia

Kubikowski, J., Lukaszewicz, J., \& Opolski, A. 1959, Acta Astron., 9, 4

Luyten, W. J. 1933, MNRAS, 93, 196

Luyten, W. J. 1997, LDS Catalogue: Doubles with Common Proper Motion (Luyten 1940-87) (Publ. Astr. Obs. Univ. Minnesota III), part 3, 35 Mason, B. D., \& Hartkopf, W. I. 2006, JDSO,2, 171M

Percival, S. M., Salaris, M., \& Groenewegen, M. A. T. 2005, A\&A, 429, 887 Rapaport, M., Le Campion, J.-F., Soubiran, C., et al. 2001, A\&A, 376, 325 Rapaport, M., , Ducourant, C., Le Campion, J. F., et al. 2006, A\&A, 449, 435

Roeser, S., Schilbach, E., Schwan, H., et al., 2008, A\&A, 488, 401

Sinachopoulos, D., \& Mouzourakis, P. 1991, A\&A, 245, 513

Taylor, B. J. 2007, AJ, 133, 370

Taylor, B. J. 2008, AJ, 136, 1388

Tian, K.-P., Zhao, J.-L., Pan, R.-S., \& He, Y.-P. 1993, ChA\&A, 17, 195

Viateau, B., Réquième, Y., Le Campion, J. F., et al. 1999, A\&ASS, 134, 173

Zacharias, N., Urban, S. E., Zacharias, M. I., et al. 2004, AJ. 127, 3043 